\title{
Influencing Factors Associated with Delayed Initiation of Insulin Among Patients with Type 2 Diabetes Mellitus at The Diabetes Clinic of a Tertiary Hospital in Ethiopia
}

\section{Elham Reshid}

School of Pharmacy, Addis Ababa University

Bruck Messele Habte ( $\sim$ bruck.messele@aau.edu.et )

School of Pharmacy, Addis Ababa University https://orcid.org/0000-0003-3866-0621

\section{Tedla Kebede}

School of Medicine, Addis Ababa University

\section{Teferi Gedif Fenta}

School of Pharmacy, Addis Ababa University

\section{Research Article}

Keywords: Delay in insulin initiation, Ethiopia, qualitative descriptive study, Tertiary Hospital, Type 2 Diabetes Mellitus

Posted Date: January 11th, 2022

DOI: https://doi.org/10.21203/rs.3.rs-1245158/v1

License: () (i) This work is licensed under a Creative Commons Attribution 4.0 International License. Read Full License 


\section{Abstract \\ Background}

Guidelines recommend the initiation of insulin in patients with type 2 diabetes mellitus who failed on maximum dose of oral medications. However, time to initiation is inconsistent due to different barriers resulting in delay and thus leads to failure to achieve glycemic control which in turn may lead to different complications. The aim of this study was to explore factors influencing the delayed initiation of insulin among patients with type 2 diabetes being managed at the Diabetes Clinic of Tikur Anbessa Specialized Hospital.

\section{Methods}

A qualitative descriptive study design was employed. Data was collected using in-depth interviews with 27 participants, including patients and healthcare providers. Audio-recorded data was transcribed and then thematically analyzed.

\section{Results}

Different factors influencing the delayed initiation of insulin in patients with type 2 diabetes mellitus were revealed. Patient factors included beliefs about the necessity of insulin and concerns related to starting insulin. Physician factors included perceived patient's situation and lack of clinical competency. Health institution factors included inadequate laboratory set up and absence of contextual guidelines for diabetes management including insulin initiation.

\section{Conclusions}

The study findings indicated different influencing factors some of which were similar to those reported from other settings while there were others which somehow were unique to study setting. These are indicative of the need to implement interventions such as strengthening the patients' diabetes health education program that is considerate of the religious, cultural, and social aspects of the society.

\section{Introduction}

According to the 2017 report from International Diabetes Federation (IDF), Diabetes Mellitus (DM) has become one of the major conditions of public health importance in developing countries that stemmed from the combined effect of globalization and epidemiological transition among others. Ethiopia is among populous African countries with the highest numbers of people with diabetes having a prevalence rate of $5.2 \%$ in 2017 (1). However, the researches done in different parts of the country already revealed a higher prevalence of diabetes up to $5.1 \%$ in 2014 (2). As the overall global picture predicts, the 
predominant type in the Ethiopian setting as well is Type 2 Diabetes Mellitus (T2DM) $(3,4)$. Metformin remains to be the mainstay therapy with a recommended shift tozh insulin therapy when treatment fails with maximum doses of Oral Anti-diabetic Medications (OAMs) (5). However, the time to initiation of insulin remains inconsistent owing, in large part, to patient, and healthcare provider factors, which presents both behavioral (lifestyle) and therapeutic challenge (4). Failure to achieve glycemic control in those patients with compromised timely initiation of insulin will result in hyperglycemia and different diabetes related complications $(3,6)$.

Evidence shows that nearly one-third of Ethiopian patients with diabetes face one or the other form of acute complications and approximately half face at least one chronic complication. According to a systematic review conducted in Ethiopia, the prevalence of neuropathy, retinopathy, and nephropathy was estimated to be approximately $35 \%, 25 \%$, and $15 \%$, respectively (7). In a similar study at the current study setting, it was found that the main causes of admission for T2DM were diabetic foot ulcer (39\%) and cardiovascular disease (21\%). Hypertension, neuropathy, nephropathy, retinopathy and diabetic foot ulcers accounted for $85 \%$ of the 756 existing complications. Overall inpatient mortality was $21 \%$ and of the 89 deaths, 77 occurred in patients with T2DM (3). Another study also reported that the direct hospital cost for patients with diabetes was significantly high and cost incurred from this to treat diabetes- related complications was reported to be significant (8).

The few studies from Ethiopia report unacceptably low levels of adherence to recommended medications as one factor compromising the successful therapeutic outcome (9). Among those, one study mentioned being on non-insulin therapy as one factor contributing to non- adherence and compromised therapeutic outcome (9). The experience of working with the local population has indicated a great deal of reluctance to commence insulin therapy among patients in the Ethiopian setting. We have in fact reported in a larger study involving 385 patients whereby $64.2 \%$ were found to have delayed initiation of insulin in their course of treatment. Those patients were left with a combination of OAMs of maximum dose instead of insulin therapy despite not achieving optimum Fasting Plasma Glucose (FPG) levels $(9,10)$. Studies have indicated that immediate initiation of insulin has led to increases in life expectancy and significant decreases in incidence and time to onset of diabetes-related complications (11). There is no study reported in the Ethiopian setting that has explored the influencing factors on the observed delayed initiation of insulin among patients with type 2 diabetes. Thus, the aim of the current study was to explore influencing factors among patients with type 2 diabetes mellitus from the perspectives of the patients, healthcare providers and the institution at the diabetes clinic of Tikur Anbessa Specialized Hospital (TASH).

\section{Methods}

A qualitative descriptive design (12) was employed to explore factors influencing delayed initiation of insulin. For the in-depth interviews with patients and healthcare providers were conducted at convenient locations such as café, hospital compound, hospital and academic offices, and other suitable work environments for the healthcare providers from January to March, 2017. Ethical approvals were obtained 
from the Institutional Review Boards of the School of Pharmacy (ERB/SOP/02/09/16) and the Department of Internal Medicine, School of Medicine (IMD/169/09), College of Health Sciences, Addis Ababa University.

\section{Study setting}

The study was conducted at the diabetes clinic of TASH, which is the largest well-organized diabetes clinic running in the country. Its clinical staffs at the time included five endocrinologists, two internists attending endocrinology fellowship program, five residents in the Internal Medicine specialty program, and five nurses. There was also a pharmacy at the clinic which constituted three pharmacists (P.C, 2016).

\section{Study participants}

Study participants were purposively selected adult patients with type 2 diabetes and healthcare providers serving these patients. Patient participants were those who had a history of delayed insulin initiation and were recruited for a larger study (10) that had a quantitative component and recruited purposively from among them. Delayed insulin initiation was operationally defined as, "patients who were on a maximum dose of two OAMs and failed to achieve optimum FPG level but not prescribed with insulin (5) due to any clinically relevant factor after three consecutive visits to the clinic of a minimum of six months duration". The additional criteria used in the larger study included age of 18 and above, being on follow up with oral medications at the diabetes clinic and switched to insulin or supposed to be switched to insulin but refused to take insulin when prescribed on the third visit. The study excluded patient charts with incomplete information regarding their oral hypoglycemic medications and their insulin initiation status. Patients with a history of gestational diabetes were also excluded. For the in-depth interview additional exclusion criteria to recruit patient participants included being healthcare providers, and not being able to orally communicate in Amharic (the official language that was also widely spoken). In this manner, 15 participants were enrolled for the study. Criteria to enroll healthcare provider participants include serving in the diabetes clinic at the time of the study. Accordingly, three endocrinologists, two internists attending the endocrinology fellowship program, two senior residents, three nurses and two pharmacists working in the diabetes clinic were enrolled for the study.

\section{Data collection methods}

For the in-depth interview, a semi-structured interview guide based on the objectives of the study and based on literature reviews (13-15) was prepared. The interview guide was then translated to Amharic. The first author conducted interviews that ranged from 30 to 60 minutes after obtaining consent from participants to be audio-recorded. Study participants were asked to discuss introductory subjects and then moved on to factors related to the patient, the healthcare providers and the health institution.

\section{Data analysis}

Interview data passed through a process of preliminary analysis whereby audio-recordings and field notes were first transcribed. Sections of original transcripts and key quotes, which were considered to be 
illustrative of the emerging themes, were translated to English. However, the process of data collection, transcription, and analysis was done simultaneously to allow a room for flexibility of the interview questions and to indicate the point of saturation. Finally, the data was analyzed using thematic analysis approach (16). Open coding was followed at the beginning where each distinct code was developed into subthemes and then themes. While developing in this procedure, previous research works $(17,18)$ were reviewed and applied while rearranging subthemes under themes.

\section{Results}

\section{Characteristics of participants}

Out of the 30 participants approached, 15 patients with type 2 diabetes and 12 healthcare providers; 7 physicians, 3 nurse, and 2 pharmacists participated in the study. Three of the patients who met the eligibility criteria did not participate for personal reasons. As shown in Table 1, most of patient participants were in the age range of 40-50 years old, female, with duration of illness less than 5 years and with more than half having no formal or primary level education. The provider participants were mostly female and had practice experiences ranging from 2 years to 20 years and had entry-level professional degree with the exception of the medical doctors as illustrated in Table 2.

\section{Thematic areas}

According to the results of the study, initiation of insulin is a process practiced by the physician who decides which patient starts insulin therapy. Nurses were found to be involved in provision of diabetes related health education at the waiting area and a one to one diabetes education and counseling sessions. The clinic at the time of the study used treatment guideline from American Diabetes Association (ADA) and IDF as a general practice but adapted to local situations of a resource-limited setting.

The clinic is using treatment guidelines from ADA and IDF but adopted to our settings. Physician, Male However, the process of initiation of insulin was not found to be uniform.

The process of initiation is not as it is expected to be. There is delayed initiation of insulin and I don't believe that there is proper initiation of insulin. Physician, Male

Participants discussed factors resulting in delayed initiation of insulin with patient, physician, and healthcare institution perspectives. 
Table 1

Profiles of the patient participants with delayed initiation of insulin interviewed at the diabetes clinic of TASH, Addis Ababa, Ethiopia, 2017. ( $\mathrm{N}=15)$

\begin{tabular}{|c|c|}
\hline Socio-demographic and other patient characteristics & Number \\
\hline \multicolumn{2}{|l|}{ Age } \\
\hline $30-40$ & 3 \\
\hline $41-50$ & 6 \\
\hline $51-60$ & 3 \\
\hline Above 60 & 3 \\
\hline \multicolumn{2}{|l|}{ Gender } \\
\hline Male & 5 \\
\hline Female & 10 \\
\hline \multicolumn{2}{|l|}{ Religion } \\
\hline Orthodox Christian & 13 \\
\hline Muslim & 2 \\
\hline \multicolumn{2}{|l|}{ Educational level } \\
\hline No formal education (can't write or read/ can read and write) & 4 \\
\hline Primary level education (1-8th grade) & 4 \\
\hline Secondary level education (9-12th grade) & 4 \\
\hline Diploma/Certificate & 3 \\
\hline \multicolumn{2}{|l|}{ Type of Job } \\
\hline House wife & 8 \\
\hline Pensioner & 1 \\
\hline Guard & 2 \\
\hline Merchant & 3 \\
\hline Company Driver & 1 \\
\hline \multicolumn{2}{|l|}{ Duration of Illness } \\
\hline$<5$ years & 5 \\
\hline $5-10$ years & 4 \\
\hline $10-15$ years & 2 \\
\hline
\end{tabular}




\begin{tabular}{|ll|}
\hline Socio-demographic and other patient characteristics & Number \\
\hline$>15$ years & 4 \\
\hline Initiated Insulin & 6 \\
\hline Yes & 9 \\
\hline No & \\
\hline Source of payment for medication & 9 \\
\hline Self-Paying & 6 \\
\hline
\end{tabular}

\section{Patient factors}

\section{Perceived adherence problems to prescribed insulin therapy}

Patients who already had adherence problem to the prescribed OAMs due to religious or stressful conditions think the same will happen with insulin. Thus, patients want to stick with OAMs with perceived better adherence.

I also think I have adherence problem and I don't think taking insulin will make any difference taking my life style into consideration. I would be happy if I can see it with oral medications of more doses. Patient, Female, 41 years 
Table 2

Profiles of the healthcare providers interviewed at the

Diabetes Clinic of TASH, Addis Ababa, Ethiopia, 2017.

$(\mathrm{N}=12)$

\begin{tabular}{|l|l|}
\hline Sociodemographic and background profiles & Number \\
\hline Sex & 5 \\
\hline Male & 7 \\
\hline Female & \\
\hline Year of practice at the diabetes clinic & 7 \\
\hline$<5$ years & 4 \\
\hline $5-10$ years & 1 \\
\hline$>10$ years & 7 \\
\hline Type of profession & 3 \\
\hline Physician & 2 \\
\hline Nurse & \\
\hline Pharmacist & 7 \\
\hline Academic qualification & 2 \\
\hline Doctor of Medicine/ Bachelor's Degree & 3 \\
\hline Internal Medicine Specialty (CSIM) & 70 (C) \\
\hline Endocrinology Sub-specialty certificate & \\
\hline
\end{tabular}

\section{Beliefs about the necessity of insulin}

Patients believe that OAMs and insulin are the same with regard to efficacy except that insulin requires complicated monitoring and management.

I have one question though. Does taking insulin really change the blood glucose level better than the oral medications? I am taking the tablets and I think I am not sick and I do not think I need to be shifted to insulin as long as I am walking healthy. Patient, F, 41 yrs. old

Patients also lack awareness on the natural course of the disease where they believe insulin is prescribed when the disease reaches advanced level. However, the present study has observed belief towards the necessity of insulin increased in patients who already started insulin. In fact, they promote timely initiation of insulin therapy as prescribed. 
I found insulin to be a good medicine now [...] I advise others to start insulin as soon as they are told. If I started insulin the day I was told, I wouldn't have faced such problem with my kidney II am having kidney problem due to the diabetes]. Patient, F, 41 yrs. old

Concerns related to starting insulin

\section{Fear of side effects and complications}

Fear of side effects like hypoglycemia and weight gain were reported to be among the reasons for patients to resist insulin treatment.

I also heard that insulin results in weight gain and I proved that right when I am taking it now. Patient, $\mathrm{F}$, 40 yrs. old

Patients also think insulin results in complications and is thus, lethal. Patients usually perceive complications as part of taking the insulin but not part of the disease process.

I know of a patient who died as a result of infection that occurred from insulin at the injection sites. I still have a concern of infection and then death. Patient, F, 41 yrs. old

\section{Fear of injections}

Patients past exposure to injections and having undergone some forms of blood taking at regular levels resulted in fear of injection.

I have a fear of needle. I even have a fear to take dust out of my eye or take a splinter out from my hands leave alone to inject myself with needle. Patient, F, 41 yrs. old

\section{Poor socioeconomic conditions}

Self-paying patients who have to buy the medication out of pocket can't afford to buy insulin and patients think shifting to insulin will incur another cost.

Definitely, insulin is expensive. It depends on the brand you are buying actually. For example, the oral medication cost them a maximum of 60 birr when they take 2 tablet 2 times a day but when we come to insulin taking the average usage of 50 unit per day, they need 2 vials which is around 112 birr. It actually is more than this since we only considered with the average usage. Pharmacist, $\mathrm{F}$

To enroll into the free government medical service, a patient is required to bring letter from the local administration (Kebele/Woreda) every four months or every year indicating they are unable to pay for their medication. Given the bureaucracy and time required to process such letters, few patients find it hard to provide support letter every month they show up at the clinic.

The hospital requires letter from Kebele every month and I could not renew my ID for a free service at Tikur Anbessa Hospital and that is my problem not to start insulin. You don't always go to Kebele begging 
that you cannot afford and it is difficult. I cannot afford the oral medicines and it is going to be more expensive with insulin. Patient, F, 48 yrs. old

\section{Perceived difficulty in insulin administration and loss of independence/reliance on others}

Older patients with visual impairments and/or those patients who cannot read or write were found to have difficulty of handling the technique of insulin injection at home. Few patients who refused to start insulin until the end of the study period do not even have someone at home who would inject them.

But, it would be impossible for me to take it with the prescribed dose [...] what if I take it with the wrong dose than the prescribed one and that might kill me... That is my problem. I am illiterate and can't read or write well.....Patient, F, 70 yrs. old

Even, some of them are frail to inject themselves despite the fact that they can read and write. I remember from my experience where patients in the same neighborhood were injecting each other with only the same dose that one patient was prescribed with. Nurse, $\mathrm{F}$

Few patients also mentioned lack of trust where same patients who are dependent on their caregivers for administration foresaw future inconvenience if caregiver support becomes diminished or compromised due to different changes.

I don't trust myself with anyone [...] you can't trust anyone; I should prepare and do it myself but my eye sights are getting weak. For example: this girl is a student and it might get late until she comes home and inject me. Patient, F, 68 yrs. Old

\section{Not amenable to religious healing (e.g. holy water use) and practices (e.g. fasting)}

Patients put down "holy water" as an alternative means to lower down their glucose level instead of shifting to insulin therapy.

I said to the doctor "I need some time". I am trying to lower the blood glucose level with "holy water". I believe it is better to lower it down with this instead of going to insulin. Patient, F, 50 yrs. old

In addition, the interference of shifting to insulin therapy with the fasting seasons was also a concern among patients following Orthodox Christian religion. Patients heard that insulin requires having proper meal and they do not think they are capable of avoiding the fasting season; they are overwhelmed with overall religious practices. This is more evident among patients of old age who are daily observant of religious practices and ceremonies. This was strengthened as follows.

I do not take my medication regularly. I go to church early in the morning and attend "mass" services. The "mass" ends around 9 am and the medicine should be taken by then. So, this is one problem for me which will be worse if I start insulin. Patient, F, 68 yrs. old 
Similarly, one patient explained that fasting decreases the blood glucose level and thus, prefer it instead of following a proper meal and uninterrupted medication schedule.

I am fasting even, the fasting decreases the glucose level and it is good. Patient, F, 70 yrs. old

One of the patients also reported on the belief where mixing religious practices, such as drinking or bathing with holy water and performing "Holy Communion", with OAMs does not get along with her religious belief. And, she thought the same will happen if she starts insulin therapy.

Religious leaders actually insist that we should take our medicines properly even if we are taking "holy water" or doing the "Holy Communion" procedure. But, I believe it is not right. It is my belief that restricts me from taking the medicines during those procedures. I don't want to mix the medicine with the body and blood of Christ. What happens if I start insulin? Patient, F, 68 yrs. Old

\section{Social factors}

One aspect of it is that patients are not willing to inject themselves when they are at work or when they are invited to weddings or friends or relatives' house thinking their friends or colleagues will stigmatize them. In addition, patients may be busy with social engagements like weddings, funerals, "Idir" (a traditional social institution to support each other in times of need, in most cases funerals), and "Iqub" (traditional saving institution). Some patients reported that insulin needs refrigeration and is not convenient to carry when moving from place to places on these occasions.

It even gets late when taking the tablet, I don't follow it properly. I might have to go somewhere early in the morning [friend's house or relative or some things I wish to accomplish]. So I take the meal and then the medicine sequentially at once thinking that it might get late by the time I get home. That is because of my tight schedule and it will be worse with insulin. Insulin needs refrigeration to carry it with me and I am not expected to take it with the meal at once, right? I need to wait some minute. So, it will be difficult. Patient, F, 41 yrs. old

Similarly, entertaining guests coming to their house was found to keep patients busy. They think insulin takes time to inject and it is against the norm and culture to stand up from the conversation having the guest sitting at home.

I may have a wedding, a funeral, or a guest might come to my place unexpectedly. Some might come to my house to seek for advice. In such cases, I cannot interrupt my conversation to take insulin since it takes time. It is against our culture to get up from a conversation. So, I do not think I can do that for the sake of taking insulin. Patient, F, 68 yrs. Old

\section{Perceived resistance to insulin}

Resistance to the medication is another concern related to insulin therapy. It is believed that patients with diabetes will through time develop resistance to insulin like the oral medications have failed to help over time. 
The main reason is that I had a fear thinking what if my body adapts to the insulin as well? It should not be stopped right? It means I am going to stop the medication if my body adapts to it and it will have no alternative medicine. That is my fear. What am I going to take after that? Patient, M, $60 \mathrm{yrs}$. old

\section{Physician barriers}

\section{Perceived Patient's Situation}

Side effects patients anticipate to occur creates sense of fear among physicians to initiate insulin therapy. The physician fears that the patient will resist or go through unexpected events, such as hypoglycemia and weight gain or might disappear from follow up if prescribed with insulin therapy. Patients also have memory problems or may not be able to read or write to initiate them on insulin therapy, according to a few physicians.

We don't tend to initiate as well if the patient doesn't fit for insulin mentally, doesn't have assistance, or have visual impairment [...]patient may have forgetting problem. Physician, $\mathrm{M}$

\section{Lack of expertise and experience}

Residents' lack expertise/lingering experience and are not comfortable or familiar to communicate with patients on the appropriate pattern of insulin initiation and titration.

There might be limited knowledge/experience on the indications. There is also lack of experience on the target FPG numbers and insulin initiation. Physician, $\mathrm{M}$

\section{Lack of motivation and confidence}

Lack of motivation and confidence among some residents (frontlines to diagnose and treat patients) who rotate frequently as part of their residency program and avoiding responsibility was found to be a factor to delay insulin.

To actually tell the truth, practically, residents are coming to diabetes clinic for short period of time as part of their rotation and they don't want to take the responsibility to initiate insulin therapy in this time. They just want to see and send the patient with the present medication he/she is taking. Physician, $\mathrm{M}$

\section{Lack of communication skill}

Physicians mainly residents lack communication skill to interact with patients regarding the disease and the medications. The communication in most cases follow a paternalistic approach where the physician dictates the patient instead of taking time to gather and provide information from and to the patient.

While initiating insulin therapy, I do a simple run down on the symptoms they will encounter and on how to treat the hypoglycemia. But the details are given by nurses. Physician, $\mathrm{F}$ 
Some of the doctors take time to explain and some do not even listen to what you are complaining let alone explaining the situation....the second ones just write your results and he/she doesn't take your points into consideration. Personality of doctors matters. The first doctor I have seen was so good to explain on initiation of insulin and titrate my dose and the second replaced physician was so fast that I couldn't catch her advices. Patient, F, 41 yrs. old

Physicians also fail to communicate well on the course of the disease and sensitize patients from the beginning on the necessity or inevitable need of insulin. In fact, insulin is considered to be a prescription as a punishment for patients who are unable to manage their blood glucose level.

The physician tells the patient that he/she will start him on insulin if the patient doesn't behave with oral medications and other factors to be considered. This means the physician is not telling the patient as insulin is imminent on the course of treatment but insulin is a punishment. Physician, $\mathrm{F}$

The physician used to tell me that I should behave with oral medications and otherwise it will be changed to the injection. May be he wants me to behave and do things right but he was trying to scare me with the injection thing at the same time. Patient, F, 38 yrs. old

\section{Health institution barriers}

\section{Absence of HbA1c test}

$\mathrm{HbA1c}$ test is not available at the hospital laboratory and the test, according to physicians interviewed, is not accessible and affordable to patients. This forces the physicians to only use FPG results to make decisions of initiating insulin therapy. Physicians, thus, demand series of records of results to decide on whether to shift to insulin therapy despite the present indications.

We advise them to take a couple of records at home for the next appointment. HemoglobinA1C is expensive for most of the patients and we usually rely on FPG. Still most of the patients can't afford to go and check their FPG. So, what you base yourself is on the thing you can have as evidence in your hand. Physician, M

\section{Lack of Continuity of Care}

Coming to contact with different physicians at different times and appointment dates, patients show lack of trust and frustration against the rotating residents prescribing the insulin therapy.

Since I started my follow up here, I was being checked up by different doctors. And, some of them are young and I am sure they are taught well to do that and I have no problem with their knowledge. But we see different face of doctors each time we come for clinic visit and it is difficult. Patient, F, $68 \mathrm{yrs}$. old
Absence of guidelines for diabetes management including insulin initiation 
The treatment guidelines and titration system used at the time in the diabetes center were designed for developed countries while the system operates in the context of a developing country in which the setup is different.

I can't say the treatment approach is uniform and that is one factor. Besides, we don't have our national guideline and we are using developed countries guideline which doesn't fit to our setting. Physician, M

\section{Lack of health educational resources}

Absence of enough teaching and learning materials prepared for the patient in local language (Amharic and others) on the general concept of diabetes and insulin presents a problem.

In my follow up at this clinic thus far, I have never encountered a patient education material prepared in Amharic. I bought one prepared material today in years. And, attention should be given to assist the patient. Patient, F, 38 yrs. old

\section{Inadequate staff for diabetes care and education}

Nurses are necessary to have a regular health education system and counseling sessions about insulin at the diabetes clinic but their low number resulted in irregular schedules at the setting.

Nurses are leaving the clinic for further education or personal reasons. And there is shortage of nurses in our setting. Sometimes, we are only two nurses to fulfill the overall activities. It is tough. Nurse, F

\section{Time barrier- High patient load and long appointment periods}

Follow up appointments are prolonged in the diabetes clinic which can extend up to six months due to high patient flow. This was difficult to follow a patient in short visits and timely prescribe insulin therapy.

In my earlier year of experiences, the number of patient seen per day was 25 and now the number is high since they cannot shift to Menelik or Ras Desta Hospital as Tikur Anbessa is known to have better supply of medicines. Nurse, $\mathrm{F}$

The appointment system is one factor. The physician has a fear of initiating insulin therapy thinking that it is impossible to see the patient in a short day appointment and follow him accordingly. The system does not allow appointing the patient whenever you want and that is a challenge to the physician to start insulin. Physician, $\mathrm{F}$

\section{Discussion}

The present study to explore the factors influencing the delayed initiation of insulin among type 2 patients is the first to be conducted in the Ethiopian setting. Findings revealed different factors related to patients, physicians and the health institution. The main findings under patient factor included the concerns about insulin fear of injection, not amenable to religious practices, and perceived difficulty of 
insulin administration. Physician factors incorporated physician's inertia where lack of clinical competency takes the priority. Health institution factors included inadequate laboratory set up and time barrier.

One of the top concern mentioned was the effect of religious practices and beliefs to start insulin therapy, which was mentioned, in another study in Ethiopia which identified religious practices as barriers to antidiabetis medications' adherence (9). The involvement of religious leaders to tackle religious concerns will improve patient's adherence as tried in the case of HIV/AIDS where religious leaders endorsed and encouraged the use of anti-HIV medications to patients while safely practicing their religious believes(20).

Perceived difficulty in insulin administration and loss of independence/reliance on others was also a finding to the present study consistent to what was documented by Lee et al. (21) where low self-efficacy presented a problem for those who cannot inject themselves or do not have someone to inject them. Thus, the involvement of family members or caregivers for the overall diabetes care or the health education session might provide substantial result.

A unique finding to the present study, however, was the perception that insulin will develop resistance as patients thought the same happened with oral medications. Considering this and almost all patient factors discussed, findings indicate the necessity of strengthening awareness program in the patient side. The present study also found that those patients who already had started insulin showed good perception about insulin.

Yet, the health education program at the diabetes clinic was found to be suboptimal where the low number of nurses was mentioned as a top reason. One method to strengthen the education will be by increasing their number. However, involving trained nurse educators would play an important role in the current setting as physicians usually refer patients to the nurses for education and instruction after prescribing insulin. This was also reported in another study where trained diabetes nurse educators can help patients to overcome psychological insulin resistance, and thus, fill the gap $(18,21)$. Moreover, the nurses should work collaboratively with pharmacist to capitalize on the education regarding insulin therapy and to focus on continued diabetes treatment and care. The impact of health education whether given in groups or in one-to-one basis is tremendous which was also stressed from a research by Habte et al. (9). But it is highly important to take into consideration patients' religious and socio-economic background.

Lack of experience and motivation was a factor according to the current findings indicating physician's inertia. This was reported in a systematic review done by Polinski et al. (22) which showed that about one-half (49\%) of the respondents reported that physicians lack experience with available types of insulin. Hence, educating patients regarding progression would take too much time. Limited experience was a key problem attached to the residents who are mostly staying at the diabetes clinic for one month as reported from the present research. The study also found that they are not motivated to start patients on insulin therapy and the decision would be referred for other residents to come for attachment or to the 
endocrinologists as also described by Lee et al. (21). This in turn was mentioned as one factor for residents to have poor communication habits in the clinic.

The type of communication, which was reported to be weak and unilateral, in most cases, was another physician factor. Although the low educational level of some patients might not allow proper interaction, low communication skill by residents and high patient flow in the system was found to affect the communication between the providers and the patients. Though this was one case, the inherent low communication skill among the residents is something that needs to be strengthened in the course of interaction with patients. The physician should develop such skills through designed communication skill trainings, seminars, and courses to create a habit to gather information from the patient and provide information or sensitize the patients about the course of treatment.

The problem of high patient flow and long appointment periods which affected the communication process was also a case accounted in a research done in Cape Town that poorly managed appointment systems and long waiting times as a result of excessive patient loads contributed to lack of continuity of care and other problems (23). The diabetes clinic is operating under the teaching and referral hospital where plenty of patients are being referred and followed in the clinic by a referral system. Patients are mostly being treated in this clinic than going to other primary healthcare and private hospitals. The reasons to this effect could be due to uninterrupted supply of insulin in the current study setting, lack of well organized health center to titrate and mange patients with insulin, and also the low socioeconomic status of the patients who can only afford public medical services.

However, the hospital along with the relevant bodies should put efforts towards adjusting the referral system by creating a path to refer well performing patients back to the health center where they are referred from. In addition, task shifting from physicians to trained nurse educators and/or pharmacists regarding dose titration might allow a room to strengthen the interaction between the patient and the physician.

Another major interaction between the institution and physician inertia, which might be unique to this study was the use of FPG instead of $\mathrm{HbA1c}$, which is in contraindication to several guidelines including that by ADA, which recommend using HbA1c (5). Thus, the decision of the physician's will be postponed and thus, delayed since series of FPG results are acquired from the patient. This is also magnified by the long patient appointment system where single result of patient's measurement taken before the appointment dates. The physician might not be certain about a single result and send the patient home with another 3 or 6 months appointment. Thus, it results in postponed decision by the physician, which in turn, results in delayed initiation of insulin. Thus, either there has to be such guidelines with local context or the system should thrive to create a well-designed system to use the available guidelines from developed countries.

Overall, the study showed the relationship among different factors, which indicated a combined intervention needed to work from the perspectives of patient, physician and health care institution to tackle the current study problem. 


\section{Conclusion}

The present study found different influencing factors that were related to delayed initiation of insulin among patients with type 2 diabetes mellitus. These factors were related to patients, physician, and the healthcare institution. Although most of the findings share similiarity to other settings, some of the findings like the religious and social factors, perceived difficulty of insulin administration, and perceived insulin resistance were found to be distinctive to the current study setting. Similarly, factors from the physician and healthcare institution were similar to other studies but lack of communication skill among the healthcare providers, inadequate labratory set up with absence of $\mathrm{HbA} 1 \mathrm{c}$, absence of national guidelines for diabetic management, low number of nurses and long appointement periods were found to be a relatively unique and important finings of the current study. The impact of health education program either in group or on one- to- one counseling sessions to address most of the problems arising from the patient is important and should be tailored to the socio-cultural and religious background of the society.

\section{Declarations}

\section{Competing interest}

The authors declare that they have no competing interest

\section{Author's contribution}

$\mathrm{ER}, \mathrm{BMH}$, and TGF were involved in the conceptualization and the design of the study. ER carried out the interviews. ER and BMH were involved in the analysis of the interviews with TK and TGF commenting on their analysis. ER drafted the manuscript and all the others revised it. All authors read and approved the final manuscript.

\section{References}

1. International Diabetes Federation. Diabetes Atlas, Brussels, Karkas Print 2017 www.idf.org/resources. Accessed on 16/08/2016.

2. Abebe SM, Berhane Y, Worku A, \& Assefa A. Diabetes mellitus in North West Ethiopia: a community based study. BMC Public Health. 2014; 14:97

3. Gizaw M, Harries DA, Ade S, Smith TA, Ali E, Firdu N, \& Yifter H. Diabetes Mellitus In Addis Ababa, Ethiopia: Admissions, complications and outcomes in a large Referral Hospital. Public Health Action. 2015; 5: 74-78

4. Owens RD. Clinical Evidence for The Earlier Initiation Of Insulin Therapy In Type 2 Diabetes. Diabetes Technology \& Therapeutics. 2013; 15:776-785.

5. American Diabetes Association (2018). Classification and Diagnosis Of Diabetes. Diabetes Care. 2018; 41:1-144 
6. Lovre D. \& Fonseca V. Benefits Of Timely Basal Insulin Control In Patients With Type 2 Diabetes. Journal Of Diabetes And Its Complications. 2015; 29:295-301.

7. Nigatu T. Epidemiology, Complications And Management of Diabetes In Ethiopia: A Systematic Review. Journal Of Diabetes. 2012; 174-180.

8. Feleke $Y$ \& Enquselassie F. Cost of hospitalization of diabetic patients admitted at Tikur Anbessa Specialized Hospital, Addis Ababa, Ethiopia. Ethiop Med J. 2007; 45:275-82

9. Habte BM, Kebede T, Fenta TG, \& Boon H. Ethiopian patients' perceptions of anti-diabetic medications: implications for diabetes education. Journal of Pharmaceutical Policy and Practice. 2017; 10:1-9

10. Reshid E. Prevalence of delayed initiation of insulin and the associated factors among patients with type 2 diabetes mellitus. [dissertation on the internet] Addis Ababa University. 2017. [cited 2017, March]. Available from: http://etd.aau.edu.et/handle/123456789/13076

11. Goodall G, Sarpong EM, Hayes C, \& Valentine WJ. The consequences of delaying insulin inititiation in UK type 2 diabetes patients failing oral hyperglycemic agents: a modeling study. BMC Endocr Disord. 2009 October; 9 (19) Available from: https://doi.org/10.1186/1472-6823-9-19s

12. Sandelowski M. Focus on Research Methods: Whatever Happened to Qualitative Description? University of North Carolina at Chapel Hill. Research in Nursing \& Health. 2000; 23:334-340.

13. Khan H, Lasker SS, \& Chowdhury TA. Prevalence and reasons for insulin refusal in Bangladeshi patients with poorly controlled Type 2 diabetes in East London. Diabetes Medicine. 2008; 25: 11081111

14. Polonsky WH, Hajos TR, Dain MP, \& Snoek FJ. Are Patients with Type 2 Diabetes Reluctant To Start Insulin Therapy? An Examination of the Scope and Underpinnings of Psychological Insulin Resistance in a Large, International Population. Curr Med Res Opin. 2011; 27(6):1169-1174.

15. Tan WL, Asahar SF, \& Harun LN. Insulin therapy refusal among type II diabetes mellitus patients in Kubang Pasu district, Kedah, Malaysia. Singapore Med J. 2015; 56: 224-227

16. Braun V \& Clarke V. Using thematic analysis in psychology, Qualitative Research in Psychology. 2006; 3(2):77-101

17. Tan AM, Muthusamy L, Ng CC, Phoon KY, Ow JH, \& Tan NC. Initiation Of Insulin For Type 2 Diabetes Mellitus Patients: What Are The Issues? A Qualitative Study. Singapore Med J. 2011; 52:801-809.

18. Ng CJ, Lai PSM, Lee YK, Azmi SA, \& Teo CH. Barriers and facilitators to starting insulin in patients with type 2 diabetes: A systematic review. International Journal of Clinical Practice. 2015; 69(10):1050-1070. doi:10.1111/ijcp.12691

19. Polosky WH, Fisher L, Guzman S, Caballero VL, \& Edelman SV. Psychological Insulin Resistance in Patients With Type 2 Diabetes: The scope of the problem. Diabetes Care. 2005; 28:2543-2545

20. Kloos H, Hailemariam D, Kaba M, \& Tadele G. Traditional medicine and HIV/AIDS in Ethiopia: Herbal medicine and faith healing: A review. Ethiopian Journal of Health Development. 2013; 27:141-55 
21. Lee KY, Lee YP, \& Jenn C. A Qualitative Study On Healthcare Professionals' Perceived Barriers To Insulin Initiation In A Multi-Ethnic Population. BMC Family Practice. 2012; 13:1471-2296

22. Polinski JM, Smith BF, Curtis BH, Seeger JD, Chowdhury NK, Connolly GJ, \& Shrank WH. Barriers to Insulin Progression Among Patients With Type 2 Diabetes: A Systematic Review. The Diabetes Educator. 2013; 39:53-65

23. Haque M, MFamMed, Navsa M, Emerson SH, Cheryl RD, \& Levitt NS. Barriers to initiating insulin therapy in patients with type 2 diabetes mellitus in public-sector primary health care centers' in Cape Town, Journal of Endocrinology, Metabolism and Diabetes of South Africa. 2005; 10(3): 94-99, DOI: $10.1080 / 22201009.2005 .10872127$ 